\title{
Revistas digitales (d)e historia medieval: panorama general Electronic journals of Medieval History: An overview
}

Resumen: La sociedad de la información ha supuesto el nacimiento de las llamadas Humanidades Digitales y esto ha hecho surgir un cambio en las publicaciones académicas dedicadas a la historia medieval. En ese contexto, nos aproximamos a un estudio de algunas revistas especializadas en el medievalismo a partir de cuatro ámbitos: a) revistas anglosajonas; b) revistas francesas; c) revistas ibéricas; c) revistas de un ámbito latino. Una vez seleccionadas las revistas, abordaremos diferentes cuestiones para su análisis, a saber: a) la indexación; b) su origen editorial; c) su formato y su relación con el OJS; d) sus directores, consejo editorial y "private army"; e) su concepto de colección; f) el sistema de evaluación; g) su contenido; h) su difusión en las redes. Por último, pasaremos a analizar los datos y los pondremos en relación con los cambios que han traído las Humanidades Digitales para comprobar los cambios y las posibles continuidades que podemos observar.

Palabras clave: Humanidades Digitales; historia digital; revistas sobre medievalismo; historiografía digital; Open Journal System.

Abstract: The information society has led to the birth of the so-called Digital Humanities and this has produced a change in those academic publications devoted to medieval history. In this context, we consider a number of publications specializing in medievalism, looking at four areas: a) Anglo-Saxon journals; b) French journals; c) Iberian journals; c) journals in the field of Latin. Having selected the publications in question, our analysis will focus on several different issues: a) their relationship with indexing; b) editorial origin; c) format and relationship with the OJS; d) directors, editorial board and their "private army"; e) their concept of collection; f) the evaluation system; g) content; h) networks of diffusion. Finally, through an analysis of the data we seek to describe and verify the changes that the Digital Humanities have made possible here, as well as the kinds of continuities that can be observed.

Israel Sanmartín, Universidad de Santiago de Compostela

2 Open Access. (C) 2019 Israel Sanmartín, published by De Gruyter. (a) BY-NC-ND This work is licensed under the Creative Commons Attribution-NonCommercial-NoDerivatives 4.0 License. 
Keywords: Digital Humanities; Digital History; Journals on Medievalism; Digital Historiography; Open Journal System.

\section{Introducción}

Las Humanidades Digitales son un campo de estudio muy joven que navega en una enorme dispersión práctica y teórica. Por el momento, está edificado en base a una tremenda indefinición. Unos lo ven como una línea de trabajo poco ortodoxa (Digital Humanities Manifesto 2.0, AA.VV 2009) y otros como una práctica (Schreibman/Siemens/Unsworth 2004: 50-100). Lentamente, las Humanidades Digitales se van convirtiendo en una disciplina académica con la creación de entidades administrativas. ${ }^{1}$ Las Humanidades Digitales se convierten en historia digital si hablamos de la disciplina de la historia. Y si existe la historia digital también tenemos que referirnos a la historiografía digital (Bresciano 2010: 33-76), concepto que empieza a aparecer en los manuales de historiografía (Poirrier 2010: 468-476). Hay algunos grupos de investigación que llevan practicando esa historiografía desde los años 90, como es el caso de la red internacional de historiadores Historia a Debate (Barros 1999: 223-242).

Por tanto, el mundo digital ha transformado la realidad académica, y han surgido la historia digital y las Humanidades Digitales, que nos lleva a preguntarnos si esas nuevas líneas de trabajo han supuesto cambios profundos en la escritura de la historia. Pero, ¿cómo ha impactado esa realidad más concretamente en las revistas científicas académicas? En ese sentido, la idea de continuidad y cambio es esencial. "Debe de haber algún punto intermedio entre quienes creen que nada puede alterar los fundamentos de nuestra labor y quienes opinan que la transformación es radical” (Pons 2013: 27).

La sociedad de la información ha supuesto, por tanto, el nacimiento de las llamadas Humanidades Digitales y la historia digital. Y esto ha hecho surgir algunos cambios en las publicaciones académicas dedicadas a la historia medieval. Con ese punto de partida, nos aproximamos al estudio de algunas publicaciones dedicadas al medievalismo en los últimos años. Y para llevar a cabo esto, haremos una división de las revistas sobre estudios medievales en cinco espacios diferentes: a) anglosajón; b) francés; c) ibérico; d) latino; y e) otras iniciativas.

1 Han surgido numerosos centros de Humanidades Digitales, como el Digital History Research Centre at University of Hertfordshire, el Virginia Center for Digital History at the University of Virginia, el Department of Digital Humanities at King's College London. 
La selección que haremos no será sistemática ni relativa a la importancia de las revistas, sino representativa de su relación ante las nuevas tecnologías y su situación dentro de la disciplina. No se trata de un análisis de las mejores revistas ni un juicio de valor sobre las elegidas. Todas son revistas de gran impacto y solvencia académica reconocida.

Una vez seleccionadas las revistas abordaremos diferentes cuestiones para su análisis, a saber: a) la indexación; b) su origen editorial; c) su formato y su relación con el OJS; d) sus directores, consejo editorial y "private army"; e) su concepto de colección; f) el sistema de evaluación; g) su contenido; h) su difusión en las redes. Por último, una vez descrita la situación de relación entre las Humanidades Digitales y las revistas de historia medieval, pasaremos a analizar los datos y los pondremos en relación con los cambios que han traído las Humanidades Digitales al mundo editorial sobre los estudios medievales.

\section{Las revistas anglosajonas}

En cuanto a las revistas de ámbito anglosajón, empezaremos por Speculum, que está asociada a The Medieval Academy of America. Veremos cómo ha realizado la conversión hacia lo digital una revista con tanta tradición. En la misma situación pero desde los años setenta, y sin estar asociada a ninguna asociación académica, está el Journal of Medieval History, del que mostraremos qué estrategia ha seguido para volcarse a internet asociándose a una de las grandes editoriales científicas. Además de estas publicaciones, hemos escogido dos novedades editoriales recientes editadas en Brill y Taylor\&Francis; se trata de Medieval Encounters y de Journal of Medieval Iberian Studies. También analizaremos la revista Digital Medievalist, que intenta romper esquemas y presenta un formato y un contexto totalmente innovador. Por último, hemos elegido a la revista Florilegium, vinculada a la Canadian Society of Medievalists, que tiene un posicionamiento más periférico espacialmente y que destaca por su hibridismo cultural (francés e inglés).

\subsection{La revista Speculum}

La revista Speculum es la primera publicación académica exclusivamente dedicada a la historia medieval. Se edita desde 1926 exclusivamente en inglés y en ella tienen cabida todo tipo de artículos interdisciplinares. En los últimos años los artículos fueron un cuarto procedentes de historia, de literatura otro cuarto, historia del arte y Byzantine studies hacen otro cuarto y un último cuarto sobre 
cuestiones metodológicas, temporales o espaciales. Destaca por una exhaustiva sección de reseñas, siempre a demanda desde la propia revista.

Su editora es The Medieval Academy of America, quien la regala a sus socios y que se ha asociado con la University of Chicago Press por espacio de cinco años, desde 2016. Hasta 2015 estaba vinculada a la Cambridge University Press, quien seguirá albergando la sede electrónica de la revista. Speculum está indexada en JSTOR, una base de datos de revistas electrónicas que se ofrece previo pago a las universidades de todo el mundo. Ofrece una lista de precios para adquirir la revista en formato electrónico o en papel y electrónico. Ofertan diferentes precios según el tamaño de la Universidad y presentan descuentos especiales para museos, bibliotecas, escuelas de secundaria y corporaciones. También ofrece la posibilidad de comprar números sueltos y disponen de un programa de descuentos y libre acceso para insituticiones educativas universitarias y de investigación de los países en desarrollo. ${ }^{2}$

La estructura de la revista, el Editorial staff, está compuesta por un Editor, un Assitant Editor, un Assitant to the Editor, un Editorial Assistant y un Copyeditor. En cuanto al Editor Board, sus integrantes son nombrados desde el Council of the Medieval Academy por un período de cuatro años bajo la recomendación del editor de la revista. Este órgano revisa los materiales enviados y recomienda los evaluadores. En su mayoría son norteamericanos. Los Review Editors son también seleccionados por el Council of the Medieval Academy, pero por un período de tres años, bajo la recomendación del editor. Su principal responsabilidad es la asignación de libros a los reseñadores y su composición está formada por académicos europeos y estadounidenses.

El sistema de revisión de los artículos sigue el clásico double-blind peer review. La revista está indexada en los principales índices mundiales como Web of Science, Arts \& Humanities Citation Index o Scopus. Exhibe que el porcentaje de aceptación de trabajos es del 9\%, el 61\% de rechazo; después hay un 10\% de revisados y vueltos a enviar, y un $20 \%$ pendiente. Esto se traduce, para el año 2014, en 20 artículos y 284 book reviews. El número de artículos ha ido variando desde los 16 de 2010; 15 en 2011; 21 en 2012 y 19 en 2013.

Los que hacen los Book reviews son expertos en los temas y son elegidos por los Review Editors. Deben de mantener un cierto criticismo pero sin caer en cuestiones personales, una cierta ecuanimidad y reflejar una clara descripción del contenido del libro y del método del autor, así como sus puntos débiles y fuertes. Les ofrecen cuatro meses para hacerlo. Pese a ello, el "Editor of Speculum reserves

2 “Chicago emerging nations initiative”, The University of Chicago Press Journals, 2018, https:// www.journals.uchicago.edu/inst/ceni [consulta 14/01/2018]. 
the right to reject reviews that do not meet the expected criteria and standards of competence and fairness". ${ }^{3}$

Los pagos de la Cambridge Universty Press a la publicación por su explotación fueron de 143.467 dólares en 2014 y 132.596 un año antes. En cuanto al dinero que les llega de JSTOR asciende a 20.144 dólares en 2013 y 26.861 en 2014. La revista siempre está atenta a las novedades y ha dedicado en 2017 (Birnbaum/Bonde/ Kestemont 2017a: 1-38) un suplemento especial a las Humanidades Digitales (Birnbaum/Bonde/Kestemont 2017b: 961-962).

\subsection{Medieval Encounters}

La revista Medieval Encounters tiene como subtítulo Jewish, Christian and Muslim Culture in Confluence and Dialogue. Se crea en 1995. Promueve la discusión interdisciplinar y comparativa entre lo judío, lo cristiano y lo musulmán desde el siglo IV al XVI. Los artículos pueden tener un contenido empírico o teórico, desde todas las disciplinas humanísticas. El editor es Ryan Szpiech, de la Universidad de Michigan, y es profesor de español allí. El review editor es de la Universidad de Amsterdam y el Consejo Editorial está conformado por profesores estadounidenses, canadienses, israelíes y algunos europeos, entre los que destacan dos españolas.

La revista pertenece a la editorial Brill y está, entre otros índices, en Scopus, Humanities International Index y ERIH PLUS. Se publican cinco números al año y los precios para instituciones son 362 euros para la versión electrónica, 398 para la versión impresa y 434 euros para la combinación de ambas. Para personas individuales el precio es de 119 euros. Usa el double-blind peer review para la evaluación de artículos, que pueden ser enviados en inglés, pero también en alemán, español o francés. Y le da a los autores la opción de publicar en Open Access, lo que implica un pago. ${ }^{4}$

\subsection{Journal of Medieval Iberian Studies}

Pertenece a la editorial Taylor and Francis, vinculada a la Western Michigan University y a la Hofstra University, y es de pago. Se crea en 2009, como una revista que publica tres números anuales con una perspectiva interdisciplinar e

3 "Guidelines for Submissions", The Medieval Academy of America, September 2017, http:// www.medievalacademy.org/?page=submission_guideline [consulta 01/02/2018].

4 “Open Access at Brill”, Brill, 2018, https://brill.com/page/OpenAccess/open-access-at-brill [consulta 03/02/2018]. 
innovadora sobre el ámbito de la Península Ibérica y sus zonas próximas, como el Magreb, Occitania, el Mediterráneo o América Latina. ${ }^{5}$ Publica textos en inglés y español que son evaluados todos con un sistema de revisión por pares (peerreview). Ofrece a los autores la opción de publicar en Open Access, previo pago. ${ }^{6}$ Está indexada en Scopus, Arts and Humanities Citation Index entre otras.

Está vinculado a la American Academy of Research Historians of Medieval Spain (AARHMS), cuyos miembros reciben la revista. El consejo editorial está encabezado por su fundador y Coordinating Editor Simon Doubleday, de la Hofstra University, de Estados Unidos. El resto de los componentes son de Estados Unidos, España y Reino Unido. La presencia internacional se acrecienta en el Advisory Board, del que, además de académicos estadounidenses y británicos, forman parte franceses, españoles, argentinos, chilenos, brasileños y portugueses. Anuncia una página de novedades sobre historia, ${ }^{7}$ así como una página Facebook y Twitter ${ }^{8}$ relativa a la historia de su subsidiaria Routledge.

\subsection{Journal of Medieval History}

Fue fundada en 1975 y se presenta como la principal revista de historia medieval en Europa. La publicación, que edita cinco números por año, funciona con el sistema de dos revisores anónimos en doble par ciego, aunque precisa que hay un initial screening que realiza el editor. Está indexada en Arts and Humanities Citation Index, Current Contents/Arts \& Humanities, y la mayoría de los índices de relevancia internacional.

Journal of Medieval History hace mención sobre la originalidad de los artículos y hace hincapié en que los artículos no pueden duplicar contenidos de otros trabajos publicados. Los editores señalan que usan el software CrossCheck para localizar los plagios. Al mismo tiempo, apunta que Taylor\&Francis facilita a los autores la opción de pagar por publicar para que los artículos se puedan consultar en acceso abierto. ${ }^{9}$

5 “Aims and scope", Journal of Medieval Iberian Studies, 2018, https://www.tandfonline.com/ action/journalInformation?show=aimsScope\&journalCode=ribs20 [consulta 02/02/2018].

6 "Open access options", Author services supporting Taylor \& Francis authors, https:// authorservices.taylorandfrancis.com/journal-list/ [consulta 04/02/2018].

7 "Explore Routledge History", Explore History on Taylor \& Francis Online, http://explore. tandfonline.com/snp/humanities\#link2 [consulta 23/02/2018]

8 Ver https://www.facebook.com/RoutledgeHistory y https://twitter.com/RoutledgeHist.

9 "Instructions for authors", Journal of Medieval History, 29/01/2018, https://www.tandfonline. com/action/authorSubmission?journalCode=rmed20\&page=instructions [consulta 23/02/2018]. 
La publicación tiene como editor al académico inglés C.M. Woolgar, de la University of Southampton. El Editorial Board está compuesto por investigadores de doce países. Por último, la revista incluye las mismas páginas de redes sociales que la revista anterior, al pertenecer a la misma editorial.

\subsection{Digital Medievalist}

Publica artículos continuamente a lo largo del año. Por tanto, tiene una estructura diferente a la tradicional. Es parte del proyecto Digital Medievalist community, enclavado en la Universidad de Lethbridge, que es una comunidad internacional que trabaja con los digital media desde el año 2003 y que abarca una revista, congresos, lista de discusión y creación de proyectos en Humanidades Digitales. Por último, está muy presente en las redes sociales ${ }^{10}$ y es de acceso abierto. Ha sido indexada en CrossRef, JISC KB+, SHERPA RoMEO y DOAJ, entre otras.

El editor es Franz Fischer, del Cologne Center for eHumanities de la Universität zu Köln en Alemania. Está respaldado por dos editores asociados de Canadá y Bélgica, mientras que el editor de las reviews es un investigador independiente. La revista funciona con el sistema de evaluación de doble par ciego, aunque en todo caso: "overall editorial responsibility rests with the journal's Editor-in-Chief, who is supported by an expert, international team of editors".11

\subsection{Revista Florilegium}

Inicia su andadura en 1979 como Florilegium: Carleton University Annual Papers on Classical Antiquity and the Middle Ages y con una idea interdisciplinar de los estudios medievales y la última Antigüedad. En 1997 se convierte en la revista oficial del Canadian Society of Medievalists/Société canadienne des médiévistes y pasa a ser editada por la University of Toronto Press. Es una revista en formato Open Access, y desde el volumen 31 se encuentra también en el proyecto MUSE, con lo que los últimos números, son de pago a través de esa plataforma. ${ }^{12}$

10 En facebook, https://www.facebook.com/groups/49320313760/, y en twitter, https://twitter. com/digitalmedieval.

11 “About”, Digital Medievalist, https://journal.digitalmedievalist.org/about/ [consulta 23/02/2018]. 12 MUSE es un repositorio sin fines de lucro en el que colaboran diferentes editoriales y la Biblioteca Milton S. Eisenhower para difundir textos y escritos académicos. Ver https://muse. jhu.edu/. 
Los idiomas de la publicación son el inglés y el francés. El sistema de evaluación es el doble par ciego que llevan a cabo tanto especialistas canadienses como internacionales. ${ }^{13} \mathrm{El}$ editor es A. E. Christa Canitz, de la University of New Brunswick. El coeditor para la parte francesa es Sébastien Rossignol (Memorial University of Newfoundland). El Editorial Advisory Board está compuesto por miembros de universidades estadounidenses, canadienses y británicas.

\section{Las publicaciones francesas}

Comenzaremos por la clásica Cahiers de Civilisation Médiévale, que se editó por primera vez en los años cincuenta y que ha tenido que abordar su integración digital en los últimos años. Otra revista con gran tradición es Cahiers d'Études Hispaniques Médiévales que, tras diferentes mutaciones, se encuentra en pleno proceso de adaptación al mundo digital. La tercera publicación escogida es $e$-Spania, que surge en los años 2000 como un proyecto innovador y asociado al libre acceso. Por último, Médiévales es una revista publicada desde los años ochenta que ha aprovechado todos los beneficios de digitalización del estado francés.

\subsection{Cahiers de Civilisation Médiévale}

Los Cahiers de Civilisation Médiévale se crearon en el año 1957 y están editados por el Centre d'Études Supérieures de Civilisation Médiévale (CESCM) de la Universidad de Poitiers. Es una revista interdisciplinar que presta especial atención a la actualidad bibiográfica publicando un gran número de reseñas. Edita cuatro números al año en los que escriben investigadores francófonos sobre la Edad Media. Anuncian que, pese a que están trabajando en una versión electrónica, la revista está alojada desde el 1958 hasta el año 2007 en Persée, que es un repositorio de revistas francesas. ${ }^{14}$ Después del año 2008, todos los números están en Carnets du CESCM aunque no en abierto, puesto que la publicación no

13 "Editorial Policies", Florilegium, https://journals.lib.unb.ca/index.php/flor/about/ editorialPolicies\#focusAndScope [consulta 23/01/2018].

14 "Persee.fr est un portail de diffusion de publications scientifiques, principalement dans le domaine des sciences humaines et sociales (...). Son positionnement est original car il assure la diffusion de collections patrimoniales (...) et il propose des outils d'exploitation similaires à ceux présents sur les portails d'édition courante (....). Ouvert en 2005, persee.fr diffuse actuellement plus de 300 collections, soit plus de 700000 documents en texte intégral et en libre accès. 
tiene versión electrónica y hay que comprarla en papel. El director es Martin Aurell, que es profesor de la Universidad de Poitiers y director del CESCM. Este cargo está apoyado por un responsable editorial, un editor de reseñas y un departamento de abonos, venta y difusión. En cuanto al comité de redacción y el consejo científico, están conformados casi en su totalidad por docentes e investigadores franceses. ${ }^{15}$

\subsection{Cahiers d'Études Hispaniques Médiévales}

Se han publicado casi cuarenta números desde su fundación en 1976. Su nombre actual se establece en el año 2005, cuando la revista se convierte en un proyecto interdisciplinar dirigido por Carlos Heusch dentro de la estructura de la École Normale Supérieure de Lyon, perteneciente a la Universidad de Lyon, que ayuda a su edición junto con las contribuciones de las suscripciones. Previamente se llamó Cahiers de linguistique hispanique médiévale, hasta que en el año 2001 nos la encontramos con el rótulo de Cahiers de linguistique et de civilisation hispaniques médiévales. Se encuentra alojada en dos repositorios: por un lado, está en Persée hasta los últimos números (2011), y también en el otro gran portal francés de publicaciones periódicas que es Cairn. ${ }^{16}$ Han escrito en ella las principales voces intelectuales sobre los temas ibéricos medievales, y su Comité Científico y su Comité de lectura están conformados por profesores franceses, ingleses y españoles, entre otros. ${ }^{17}$

\section{3 e-Spania}

e-Spania. Revue interdisciplinaire d'études hispaniques médiévales et modernes es editada por el CLEA (Civilisations et Littératures d'Espagne et d'Amérique du

Le portail accueille en moyenne 30 millions de visites par an”. “À propos de Persée”, http://www. persee.fr/a-propos [consulta 20/11/2017].

15 “Cahiers de Civilisation Médiévale”, Université de Poitiers, http://cescm.labo.univ-poitiers.fr/ publi/cahiers-de-civilisation-medievale/ [consulta 10 de dicembre de 2017].

16 "Cairn.info nació del interés de cuatro editoriales vinculadas a las Humanidades y a las Ciencias Sociales (Belin, De Boeck, La Découverte y Erès) para tener mayor presencia en Internet. En el año 2006 se unió la Bibliothèque nationale de France para impulsar el proyecto editorial francófono en red, aunque también gestionan las versiones en papel”, "À propos de Cairn.info", Cairn.info, https://www.cairn.info/a-propos.php [consulta 4 de febrero de 2018]

17 “Cahiers d’Études Hispaniques Médiévales”, https://www.cairn.info/en-savoir-plus-sur-larevue-cahiers-d-etudes-hispaniques-medievales.htm [consulta 04/02/2018]. 
Moyen-Age aux Lumières) dos veces al año. A partir del año 2014 cambiaron a tres números (febrero, junio y octubre). Comenzó su publicación en el año 2006 con una orientación transdiciplinar y una proyección internacional. En su presentación justifica la elección del formato digital por su difusión, gratuidad y practicidad.

El Comité de lectura y el Comité científico se preocupan del buen gobierno y de la proyección internacional de la revista, que tiene un promedio de más de 10.000 visitas al mes. El fundador y director honorario es Georges Martin, de la Universidad Paris-Sorbonne, igual que su directora Hélène Thieulin-Pardo.

En cuanto al funcionamiento, concretan que los artículos enviados serán evaluados “anonymement à deux expertises confiées à des membres du comité de lecture, du comité scientifique ou à des personnalités extérieures". ${ }^{18}$ Por último, la revista está indexada en el DOAJ, Base (Bielefield Academic Search Engine), EZB (Elektronische Zeitschriftenbibliothek), o Journal TOCs.

\subsection{Revista Médiévales}

Existe desde 1982 y está editada por las Presses Universitaires de Vincennes. Está especilizada en la temática medieval desde un ámbito interdisciplinar y se organiza a partir de un dossier temático, aunque hay artículos sueltos, recensiones, etc. El comité de redacción examina colectivamente todos los artículos y luego el consejo científíco internacional es consultado "pour avis sur les articles relevant de leur domaine de compétence. Les ouvrages pour comptes rendus doivent être adressés à la rédaction”. ${ }^{19}$

La dirección corresponde a Laurence Moulinier-Brogi, profesora de la Université Lumière Lyon 2; Christopher Lucken (Université Paris 8 VincennesSaint-Denis) es el redactor jefe con Danièle Sansy (Université Le Havre Normandie). El Comité de Redacción está integrado por académicos franceses y el Consejo Científico está prácticamente en manos de profesores e investigadores franceses también. Médiévales tiene una versión impresa y una electrónica, que está alojada en el proyecto editorial francés OpenEdition. ${ }^{20}$ Oferta sus últimos tres números en el portal Cairn y son de pago; el resto de la colección de la revista está

18 “Contributions - Expertises”, e-Spania, http://journals.openedition.org/e-spania/18355 [consulta 04/02/2018].

19 "La revue”, http://journals.openedition.org/medievales/866 [consulta 14 de febrero de 2018].

20 "Missions", OpenEdition, http://www.openedition.org/6438 [consulta 14/02/2018]. OpenEdition está desarrollada por el OpenEdition Center, la Unité de service et de recherche CNRS, de l'université d'Aix-Marseille, del l'EHESS y de la Université d'Avignon. 
en la página hasta el año 2002. Hasta ese año desde el número uno, publicado en 1982, están alojados en Persée.

\section{Revistas ibéricas}

El tercer espacio que hemos seleccionado es la Península Ibérica, España y Portugal. En ese ámbito hemos escogido el Anuario de Estudios Medievales, que ha hecho un gran trabajo de volcado al mundo digital en formato abierto. Además del Anuario, hemos analizado la revista Imago Temporis: medium Aevum, que ha surgido en los últimos años como una de las representantes de la nueva historiografía medieval española, donde prima la internacionalización y la interdisciplinariedad. La tercera revista elegida es la vinculada a la Sociedad Española de Estudios Medievales, Medievalismo, que está en pleno proceso de digitalización. Por último, la publicación portuguesa Medievalista nace como nativa digital y ha alcanzado una especial visibilización en el campo académico del medievalismo internacional.

\subsection{Anuario de Estudios Medievales}

Fue fundado en el año 1964. Se trata de una publicación anual que pertenece al conjunto de revistas que edita el CSIC. Se publica en dos fascículos, que buscan recoger trabajos sobre el Medievo desde diferentes disciplinas y enfoques. Los idiomas en que se publica son: castellano, catalán, portugués, francés, italiano e inglés. Se encuentra indizada en numerosas bases de datos nacionales e internacionales, como la Web of Science, Arts \& Humanities Citation Index o SCOPUS, entre otras de prestigio internacional.

Está dirigido por Roser Salicrú, del Instituto Milá y Fontanals del CSIC. Además, tiene una Secretaría y un Consejo de Redacción, formado por trece profesores españoles, un alemán y un suizo. En cuanto al Consejo Asesor, lo forman quince españoles, tres estadounidenses, dos italianos, dos franceses, dos portugueses, un griego y un británico. Los artículos son evaluados por el sistema de evaluación por pares y defiende la política de acceso abierto, aunque es posible adquirir la revista en papel (sobre 100 euros en España y 130 en el extranjero). Y, además, añaden que tienen una política antiplagio.

Incluye un listado de evaluadores (que previamente han autorizado su inclusión en la lista) y un resumen de su actividad editorial. La última que ofrece es del 2015-2016, cuando certifican que recibieron 58 artículos en 2015, 
de los cuales fueron rechazados un 59\%, cifra que aumentó hasta el 76\% al año siguiente con una recepción de 41 trabajos.

\subsection{Imago Temporis: Medium Aevum}

La revista Imago Temporis surge en el año 2007 para contribuir a la renovación de los estudios medievales, con especial relación a los países del área mediterránea, con un carácter interdisciplinar. Surge gracias a la iniciativa del profesor Flocel Sabaté y del Grup de Recerca Consolidat en Estudis Medievals Espai Poderi Cultura de la Universidad de Lleida. Se inicia en formato papel, aunque después se ha transformado también en digital bajo el formato OJS. Está indexada en Carhus $+\mathrm{y}$ forma parte del repositorio denominado "Revistes Catalanes amb Accés Obert. ${ }^{21}$ La publicación está indexada en el ISI Web of Knowledge y en las otras bases de datos internacionales. Está publicada en inglés ${ }^{22}$ y el Scientific board está conformado por medievalistas de veintiocho países. Por su parte, el Editorial board está compuesto por historiadores de doce estados.

\subsection{Medievalismo}

Nace en el año 1991 y se edita gracias al amparo de la Sociedad Española de Estudios Medievales y del Servicio de publicaciones de la Universidad de Murcia. Tiene una periodicidad anual. Está indexada en el Emerging Sources Citation Index, o ERIH PLUS, entre otros. Además, tiene el sello de calidad de la FECYT. $\mathrm{Su}$ directora es la profesora María Isabel del Val y los secretarios de redacción son Francisco García Fitz y Juan F. Jiménez Alcázar. El Consejo de Redacción está configurado por académicos españoles, franceses, italianos, estadounidenses y argentinos. Por su parte, el comité científico tiene en nómina a académicos de diferentes países americanos y europeos. Surgió como una revista en papel y ahora se encuentra ya en formato OJS, alojado en el servidor de la Universidad de Murcia. Tiene una importante sección de reseñas.

21 “Què és?”, Revistes Catalanes amb Accés Obert, http://www.raco.cat/index.php/raco/pages/ view/QueEs [consulta 15/01/2018].

22 "Journal information", Imago Temporis, http://www.medieval.udl.cat/en/imago/Journalinformation/ [consulta 15/01/2018]. 


\subsection{Revista Medievalista}

Se funda en el año 2005, gracias a la iniciativa del profesor Luis Krus, dentro del Instituto de Estudos Medievais de la Faculdade de Ciências Sociais e Humanas da Universidade Nova de Lisboa, para llenar el vacío de las revistas sobre historia medieval en Portugal. Hacen hincapié en el carácter interdisciplinar y en su propósito de "promover e divulgar novas abordagens ao estudo da Idade Média". ${ }^{23}$

El director es Bernardo de Vasconcelos, quien está respaldado por nueve editores de diferentes universidades portuguesas, fundamentalmente de Lisboa. En cuanto al Consejo Editorial, está conformado por profesores de nueve países. Está indexada en Latindex, Dialnet, Revues.org, DOAJ, ERIH PLUS, y SciELO. También se publican todos los evaluadores de los artículos desde el año 2010.

\section{Revistas latinas}

El último ámbito espacial que hemos acotado lo hemos denominado como "latino". Ahí han surgido dos revistas nativas digitales ancladas en España pero desarrolladas en Brasil. Se trata de Mirabilia Journal y Roda da Fortuna. La tercera revista es Signum, que es el medio de comunicación de la ABREM. Por último, hemos seleccionado Reti Medievali, que es una de las revistas emergentes en los últimos años desde un ámbito latino.

\subsection{Mirabilia Journal. Electronic Journal of Antiquity, Middle \& Modern Ages}

Mirabilia Journal es una revista editada en el marco del Institut d' Estudis Medievals de la Universidad Autónoma de Barcelona. En su concepción tiene un enfoque hacia la historia cultural. ${ }^{24}$ Ha publicado 25 números y 431 artículos. El director de honor es José E. Ruiz-Domènc (UAB), y la dirección la comparten Adriana Zierer (Universidade Estadual do Maranhão) y Moisés Romanazzi Tôrres (Universidade Federal de São João del-Rei). El Editorial board está integrado por académicos brasileños y algún español. Por su parte, el Board Policy está

23 "Medievalista online revista especializada em Estudos Medievais", Medievalista online, http://www2.fcsh.unl.pt/iem/medievalista/ [consulta 01/03/2018].

24 “About", Revista Mirabilia, http://www.revistamirabilia.com/about [consulta 14/02/2018] 
compuesto por integrantes de universidades brasileñas, fundamentalmente, con alguna presencia española, alemana y estadounidense.

La revista funciona con el sistema de evaluación de external Double Blind Peer Review Process, aunque especifican que los editores evalúan primero los manuscritos y rechazan aquellos que no son originales o no son serios en el lenguaje o en el uso científico. La revista tiene una política de acceso abierto.

\subsection{La revista Roda da Fortuna}

Es una publicación interdisciplinar que tiene como objetivo publicar textos relacionados con la Antigüedad y el Medievo. La evaluación de sus trabajos se realiza bajo el sistema doble par ciego y no tiene ánimo de lucro, por lo que exhibe una política de acceso abierto. Las líneas de investigación son muy concretas: "Cultura, Memória e Narrativas na Antiguidade e Medievo” y "Poder e Relações sociais na Antiguidade e Medievo”. 25

Los directores de la revista son Guilherme Queiroz de Souza (Universidade Federal da Paraíba) y el Dr. Luciano José Vianna (Universidade de Pernambuco). El Consejo editorial está integrado por investigadores de universidades de Brasil, Italia, Argentina, España, Reino Unido y Estados Unidos. El Consejo Directivo, en su conjunto, tiene la decisión final de la publicación de los artículos después de su evaluación. La revista está indexada como “B2” por el CAPES (Coordenação de aperfeiçoamento de pessoal de nivel superior), que es una fundación brasileña que depende del Ministerio de Educación y que, entre otras cuestiones, se dedica a la evaluación de las publicaciones brasileñas, además de ser un repositorio.

\subsection{Signum}

La revista Signum es una revista de historia medieval creada en 1996, que pertenece a la ABREM (Associação Brasileira de Estudos Medievais) y tiene la calificación “B1” por la fundación Capes para el Área de Historia, aunque también está indexada en el Sumário de revistas brasileñas y otras bases de datos.

El equipo editorial está encabezado por los dos editores principales, Carolina Coelho (Universidade Federal Fluminense) y Paulo Duarte (Universidade Federal do Rio de Janeiro). Estos están acompañados por una asistente editorial y un

25 "Quem somos", Revista Roda da Fortuna, https://www.revistarodadafortuna.com/quemsomos [consulta 14/02/2018]. 
conjunto de editores (historia, arte, literatura, filosofía y derecho). El Consejo editorial está conformado por profesores de Portugal, Suiza, Brasil, Colombia y Argentina. Por último, el Consejo científico está integrado por investigadores de Francia, Estados Unidos, Brasil, Argentina, Portugal y España

La sede de la revista es itinerante, puesto que depende de la dirección de la ABREM. Se publicó en formato impreso entre 1996 y 2010, año en el que se adoptó el formato digital. Es una revista que publica dos números al año y tiene carácter interdisciplinar. ${ }^{26}$ Recibe artículos en portugués, inglés y español, y publica artículos inéditos, dosieres, reseñas y traducciones "produzidos por doutores”. La revista tiene una política de acceso libre. Por último, tiene una página de Facebook con casi quinientos seguidores.

\subsection{Reti Medievali}

Es una publicación que nace en 1998, a partir de un grupo de académicos italianos, para responder a la fragmentación historiográfica. Opta por el formato digital para disfrutar de las ventajas de acceso y difusión de la red. Está editada por la Firenze University Press con el formato Open Journal System y publicada en la Università di Napoli Federico II. Todos los artículos son evaluados por el sistema de doble par ciego. Asimismo, la Agenzia Nazionale di Valutazione del sistema Universitario e della Ricerca (ANVUR) la ha calificado como "fascia A", el nivel más elevado en su área. Además, está indexada en el Arts and Humanities Citation Index, ERIH PLUS y SCOPUS. ${ }^{27}$

La coordinación de la revista está en manos de cuatro académicos de universidades italianas: Università degli Studi di Napoli Federico II, Università degli Studi di Genova, Università degli Studi di Verona y la Università degli Studi di Torino. Está conformada por veintidós Redattori, fundamentalmente de Italia, aunque también hay un alemán, un francés y un español. Además, tiene una serie de Redattori Corrispondenti con profesores franceses, alemanes, italianos, argentinos, ingleses y españoles. Y, por último, un Comitato scientifico compuesto por italianos, estadounidenses, franceses, alemanes. Aplica un sistema antiplagio y en el 2002 se refuerza con la constitución de la Associazione Culturale Reti Medievali, para favorecer el estudio y la investigación de la historia medieval.

26 "Políticas Editoriais", Revista Signum, http://www.abrem.org.br/revistas/index.php/signum/ about/editorialPolicies\#focusAndScope [consulta 14/02/2018].

27 "Politiche editoriali", Reti Medievali, http://www.rmojs.unina.it/index.php/rm/about/ editorialPolicies\#focusAndScope [consulta 23/01/2018]. 


\section{La historiografía digital y el modelo anglosajón}

Lo que vamos a analizar a continuación es la confluencia de la mayoría de las revistas hacia el modelo de la Web of Science (WOS), que es una plataforma que recoge las referencias de las principales publicaciones científicas de cualquier disciplina del conocimiento desde 1945. Esta plataforma ha servido para crear un sistema favorable a la cuantificación del saber científico y, como consecuencia, a la indexación de las revistas, que se miden en relación con su presencia en índices de calidad, como el Arts \& Humanities Citation Index, Scopus, ERIH PLUS, etc. Así, las publicaciones periódicas han tenido que adaptarse a una nueva realidad de calidad y estandarizar sus protocolos de publicación y de organización. Lo que hemos mostrado hasta aquí es cómo lo han hecho diferentes revistas nativas en papel y otras en formato digital. Veamos ahora qué consecuencias tiene esto.

En el ámbito anglosajón hemos analizado en primer lugar la revista Speculum, que pese a su longevidad se ha adaptado al mundo digital con gran solvencia. Su pertenencia a la Medieval Academy of America la ha mantenido como un instrumento de gran prestigio. La revista comenzó siendo en papel pero se volcó al mundo digital a través de la plataforma JSTOR. Por tanto, ha hecho una transición de pago de papel a pago en digital, manteniéndose como una revista de gran valor simbólico. Este ha sido reforzado por el prestigio de pertenecer a sus comités y por su alta "selectividad" en la revisión de artículos, que se hace bajo el sistema de doble par ciego. Su concepto de colección ha cambiado significativamente, puesto que ha evolucionado del concepto de colección física a digital o, incluso, a la posibilidad de consultar y poseer artículos sueltos. Su difusión en las redes es a partir de la web de la Medieval Academy of America y es restringida.

Siguiendo el modelo de Speculum está la revista Florilegium, que pertenece a la Canadian Society of Medievalists. Ofrece gran parte de sus números en acceso abierto y sólo los últimos a partir del proyecto MUSE en formato de pago.

La revista Medieval Encounters pertenece a la editorial Brill, lo que la indexa automáticamente en los principales índices internacionales. Se edita en formato impreso y digital, ambos de pago, con lo que no hay posibilidad de acceso abierto a los trabajos, salvo que el autor pague una cantidad. Los componentes de los diferentes comités de la revista son profesores de relevancia internacional, que certifican una evaluación por pares y garantizan una rigurosidad en los contenidos. Por último, su difusión en las redes está mediatizada por ser de pago.

El Journal of Medieval Iberian Studies es una revista estadounidense que pertenece a la editorial Taylor and Francis y figura en los grandes índices 
internacionales. Su origen editorial es digital y está vinculada a la American Academy of Research Historians of Medieval Spain. En otro sentido, su director y los miembros de sus consejos están mediatizados por el "subject” de la revista, que es el mundo medieval ibérico. Es de pago y su difusión en las redes está limitada por ser de pago, y se realiza a partir de las páginas de Routledge, aunque publican artículos en abierto previo pago de una cuota económica.

Muy en la línea de las anteriores está el Journal of Medieval History, puesto que pertenece también a la editorial Taylor and Francis, lo que le garantiza un posicionamiento perfecto en los principales índices mundiales. De origen británico, surge como una revista en papel que hizo una transición ejemplar al mundo digital, conservando la esencia de ser de pago. Su concepción de colección se ha ido fragmentando a medida que se ha ido digitalizando. El sistema de evaluación ofrece todas las garantías del sistema de doble par ciego y su difusión en las redes es la propia de la editorial.

Por último, Digital Medievalist es una revista nativa digital con un carácter innovador, puesto que rompe con el concepto de "número de revista" y de colección para introducir un "continuum" de artículos a lo largo del año. De tal forma, los artículos se van publicando a medida que se aprueban. No está indexada en los principales índices internacionales y sus editores y consejo editorial pretenden colaborar en la reflexión sobre el mundo digital en relación con el medievalismo. Están vinculados a una comunidad digital llamada "Digital Medievalist Community".

Por tanto, se advierte que las revistas anglosajonas están incluidas en los principales índices; que su publicación suele estar vinculada a grandes editoriales académicas; que son de pago y en formato "cerrado"; que el concepto de colección se ha visto alterado por su carácter pecuniario; que el sistema de evaluación es el de doble par ciego; que sus contenidos están vinculados a temas que se consideran tópicos internacionales de investigación; y que su difusión en las redes es limitada. Sólo el caso de Digital Medievalist rompe con esa dinámica.

Cahiers de Civilisation Médiévale no muestra un especial interés por estar presente en los principales índices de evaluación. Su origen editorial es en papel y en estos momentos están todavía haciendo la transición hacia la versión digital, aunque han colgado en el repositorio francés Persée una gran parte de su producción editorial con acceso gratuito. La revista es de pago y los últimos números están en acceso solo previa cotización. Pretende continuar con un concepto de colección tradicional pese a la traslación al mundo digital. Es una publicación integrada fundamentalmente por académicos franceses que ejercen un control riguroso de los artículos. Su difusión en la red está lastrada por figurar en varios sitios web. 
Si la anterior revista está ligada a Poitiers, los Cahiers d'études hispaniques médiévales están vinculados a Lyon y a una publicación en papel. Tampoco hay una especial preocupación por los índices internacionales anglosajones y está conformada por académicos franceses, ingleses y españoles. El sistema de evaluación también está en manos de los comités correspondientes, y su difusión en las redes se encuentra por un lado en el repositorio Persée, que ofrece gratuitamente los números de las revistas hasta 2011. A partir de esa fecha, la revista se encuentra en el portal Cairn, que es de pago.

e-Spania. Revue interdisciplinaire d'études hispaniques médiévales et modernes se encuentra indexada en índices fundamentalmente europeos. Es una revista nativa digital con formato OJS, editada desde Francia con proyección internacional (en sus contenidos y diferentes consejos editoriales) y con un sistema de evaluación anónimo. Hacen gala de su gratuidad, y su circulación a partir de su página web tiene un notable éxito.

Por otro lado, Médiévales es una publicación que sigue las grandes líneas de las dos primeras revistas francesas analizadas en cuanto a indexación, origen editorial y consejo editorial. La única novedad es su vinculación con otro de los grandes proyectos públicos franceses de difusión de la cultura científica de ese país, que es OpenEdition, donde se pueden encontrar todos los números desde el año 2002. Los anteriores están en Persée y los últimos en Cairn, previo pago de su consulta.

En conclusión, en las revistas francesas hay una despreocupación por la indexación anglosajona así como por todo el sistema de evaluación del par ciego. $\mathrm{Su}$ publicación está vinculada a grandes editoriales universitarias públicas y usan los diferentes repositorios públicos para ofrecer en acceso abierto el contenido de números antiguos. Los últimos números son de pago y su difusión en las redes es una prioridad para los editores, aunque no en las páginas propias de las revistas, sino en los repositorios. La revista e-Spania es la más innovadora y, salvo la gratuidad total, sigue el modelo de las anteriores.

En cuanto a las revistas ibéricas, el Anuario de Estudios Medievales está indexado en los principales índices. Su origen editorial es en papel pero han hecho un volcado modélico a su versión en Internet y en acceso abierto. Esto no ha alterado la idea de colección de la revista, que conserva su estructura con un interface gráfico muy claro y elaborado. El personal de la revista tiene como base España, pero con algunos profesores de otras historiografías, y el sistema de evaluación es el de doble par ciego. Su difusión en las redes es ejemplar, con una página muy accesible y muy navegable. Muestran una transparencia máxima con la publicación de los evaluadores y los últimos números se pueden comprar en formato papel.

Imago Temporis: medium Aevum sigue en líneas generales la revista anterior, con la peculiaridad de que la revista en formato digital tiene una elaboración 
mucho menor. Tiene una internacionalización absoluta en todos los órganos colectivos. Continuando en el espacio ibérico, Medievalismo está referenciada en índices internacionales, aunque no al nivel de las anteriores. El interface de la revista en acceso abierto está todavía en construcción. Su característica diferenciadora es que es la publicación de la Sociedad Española de Estudios Medievales, que también la edita en papel.

Por último, la revista Medievalista es una publicación portuguesa que nace en formato digital y que se referencia a todo el sistema métrico y de clasificación anglosajón en cuanto a funcionamiento colectivo e indexación. Es una publicación gratuita, con una apariencia muy atractiva estéticamente y buen funcionamiento, con un alto interés en la transparencia de los procesos de calidad.

Por tanto, en las revistas ibéricas hay una preocupación por la indexación anglosajona y por vincularse a ese modelo del sistema de calidad internacional, así como por toda la metodología sistemática de evaluación del par ciego. Su publicación está vinculada tanto a instituciones públicas (Universidad, CSIC) como a sociedades de medievalistas. Las revistas se ofrecen en su formato digital de forma gratuita, y las páginas webs están todavía en construcción.

En cuanto a las revistas latinas, Mirabilia es una revista nativa digital brasileña, que busca las indexaciones y el funcionamiento implantado por las revistas anglosajonas. Su concepto de revista es de acceso abierto con una apuesta muy clara por su difusión en Internet y en las redes sociales. En una situación muy similar está la revista Roda da Fortuna, aunque con una mayor internacionalización en sus comités. Ambas tienen una web propia y una alta calificación en el sistema brasileño de calidad coordinado por el CAPES.

Una indexación alta en el sistema brasileño lo tiene también la revista Signum, que pertenece a la Associação Brasileira de Estudos Medievais, que es lo que la distingue de las dos anteriores. Además, esta revista nació como una publicación impresa y luego ha hecho el volcado a la versión on line. Permite artículos en diferentes idiomas y tiene una página en Facebook.

Para terminar, Reti Medievali es una revista italiana que se vincula también a los grandes índices internaciones y a una creciente internacionalización en sus órganos colegiados. Nace como una revista defensora del modelo digital y del formato Open Journal System. Ofrece también un gran esfuerzo por mostrar transparencia en su funcionamiento.

De esta forma, en las revistas que hemos denominado como "latinas" se detecta, como en las ibéricas, que hay una preocupación por la indexación anglosajona y por la asociación a un sistema de medición donde priman los valores cuantitativos. Su publicación está vinculada tanto a instituciones públicas, como a sociedades, y se difunden a partir de páginas webs y redes sociales. La esencia de su funcionamiento es el acceso abierto a sus contenidos. 


\section{Conclusiones}

Una vez descrita la situación de relación entre las Humanidades Digitales y las revistas de historia medieval, pasaremos a analizar los datos y los pondremos en relación con los cambios que han traído las Humanidades Digitales al mundo editorial sobre los estudios medievales:

a) Podemos concretar dos características importantes en las revistas analizadas: la digitalización y la búsqueda de una adecuación al modelo anglosajón de ciencia con las indexaciones y el funcionamiento de las revistas con evaluaciones y comités internacionalizados.

b) Las continuidades con respecto a las revistas tradicionales tienen que ver con la temática, las ediciones en papel y su conexión con editoras públicas o privadas.

c) No han supuesto un cambio visible en la forma de escribir la historia, pero el sistema ha variado la forma de escribir los artículos. Ahora se realizan para revistas indexadas que tienen que pasar una serie de estándares de calidad. Hay una mayor traducción al inglés de los trabajos; hay una búsqueda de agrado más evidente y una mayor publicación de artículos debido a las demandas del sistema.

d) Sehaacabadola revistatradicionalen el sentidodel papel, en su funcionamiento y en su ejecución. Ahora las revistas buscan indexarse y tener visibilidad en la red.

e) Han surgido nuevas revistas que, además, pueden llegar a los índices de gran calidad, sobre todo si están relacionadas con grandes editoriales, pero no han destronado a las clásicas, que han buscado su acomodo en la nueva situación.

f) La evaluación por pares ha supuesto una revolución en la calidad de las revistas. Así, la legitimación de la ciencia histórica se produce mediante la generalización de un método, por la institucionalización de la escritura y por la relación de las narraciones históricas con el mercado.

Más en concreto podemos decir que, en relación con la indexación, las revistas anglosajonas asociadas a grandes editoriales buscan referenciarse en los índices y no así los nuevos proyectos de revistas digitales. Las revistas ibéricas y latinas que hemos analizado siguen este modelo anglosajón de indexación para catalogarse como revistas de calidad y recibir más trabajos de mejor valoración. Diferente es el modelo francés, el cual se ha desarrollado de forma más independiente de esos índices y valores cuantitativos.

En cuanto a su origen editorial, las revistas anglosajonas están vinculadas a editoriales privadas o a sociedades de historiadores, y son en su mayoría de 
pago. Las del mundo francés están vinculadas a universidades públicas o a centros de investigación, y guardan una relación híbrida entre ofrecer gratis los contenidos de años pasados y de pago los más actuales. Los modelos ibéricos y latinos, al ser revistas de nueva creación, se ofrecen fundamentalmente en sistema de acceso abierto, y sus editoras son esfuerzos de equipos asociados a los servicios de publicación de diferentes universidades.

En relación con el Open Journal System, todas aquellas revistas que se ofrecen en este sistema suelen ser gratuitas; las de pago están asignadas a las páginas editoriales. En esta situación son una excepción las francesas, que están alojadas en grandes repositorios estatales tanto gratuitos como de pago.

Los Consejos Editoriales y Científicos de las revistas han evolucionado hacia una gran internacionalización, el sistema de evaluación es el de doble par ciego, menos en el modelo francés que lo dejan en evaluaciones anónimas. Todavía las revistas siguen avanzando en sus páginas webs y en las redes sociales.

En definitiva, lo nuevo se entiende mejor exponiendo lo viejo. Lo digital no supone dejar a un lado lo tradicional (Cohen/Rosenzweig 2006: 10-100). Por el contrario, supone la multiplicación de la información y de los proyectos, pero también representa la posibilidad de un cambio en la escritura de la historia y en la mutación hacia nuevos formatos (Gallini/Noiret 2011: 16-37). Paralelamente, también ha cambiado la lectura y su relación con la escritura, que son los elementos centrales de nuestro oficio.

Es un debate sobre la relación entre la historia y la tecnología (Canaparo 2003: 45-50). En ese sentido, debemos de seguir discutiendo sobre aspectos como: ¿qué sucede con el concepto de “colección completa” de una revista?, ¿sigue existiendo el de línea editorial de una publicación periódica?, ¿sigue teniendo sentido el equipo de redacción?, ¿cuáles son las diferencias entre la versión impresa y la digital? Pero nos quedan otros muchos: ¿sigue existiendo el concepto de portada?, ¿qué pasa con la idea de suscriptor? En el mismo sentido, ¿podemos incluir esta historiografía digital dentro de lo que se denomina el pluralismo interpretativo de la historiografía desde los años 2000? (Delacroix/ Dosse/Garcia 2005: 578-633). Y, por último, ¿se disolverá la historia digital en la llamada Historia pública? (Pons 2010).

\section{Referencias Bibliográficas}

AA.VV. (2009): “Digital Humanities Manifesto 2.0”. En: ucla.edu 29 mayo. [En línea, http:// manifesto.humanities.ucla.edu/2009/05/29/the-digital-humanities-manifesto-20/, consulta 22/06/2017]. 
AA.VV. (2016): “Manifeste des Digital Humanities”. En: hypotheses.org, 26 de marzo. [En línea, http://tcp.hypotheses.org/318, consulta 22/06/2017].

AA.VV. (2001): Manifiesto historiográfico historia a Debate. Santiago de Compostela: Historia a Debate.

Barros, Carlos (2001-2002): “Defensa e ilustración del Manifiesto historiográfico de Historia a Debate”. En: Revista d'Història Medieval, 12, pp. 389-433.

Barros, Carlos (1999): “Hacia un nuevo paradigma historiográfico”. En: Memoria y civilización, 2, pp. 223-242.

Birnbaum, David J./Bonde, Sheila/Kestemont, Mike (2017a): “The Digital Middle Ages: An Introduction”. En: Speculum, 92.S1, pp. S1-S38.

Birnbaum, David J./Bonde, Sheila/Kestemont, Mike (2017b): “The Digital Middle Ages. A Speculum Supplement”. En: Speculum 92.4, pp. 961-962.

Bresciano, Andrés (2010): La historiografía en el amanecer de la cultura digital. Montevideo: Ediciones Cruz del Sur.

Canaparo, Claudio (2003): Ciencia y escritura. Argentina: Zibaldone.

Cohen, Daniel J./Rosenzweig, Roy (2006): Digital History: a Guide to Gathering, Preserving, and Presenting the Past on the Web. Philadelphia: University of Pennsylvania Press.

Delacroix, Christian/Dosse, François/Garcia, Patrick (2005): Les courants historiques en France. $X I X-X X$ siècle. Paris: Gallimard.

Gallini, Stefania/Noiret, Serge (2011): “La historia digital en la era del Web 2.0. Introducción al dossier Historia digital”. En: Historia Crítica, 43, pp. 16-37.

Pons, Anaclet (2013): El desorden digital. Guía para historiadores y humanistas. Madrid: Siglo XXI.

Pons, Anaclet (2010): “La historia digital y la historia pública." En: Clionauta: blog de historia 25 de junio. [En línea, https://clionauta.wordpress.com/2010/06/25/la-historia-digital-yla-historia-publica/, consulta en 13/01/2018].

Poirrier, Philippe (2010): “Internet et les historiens”. En: Delacroix, Christian/Dosse, François/ Garcia, Patrick/Offenstadt, Nicolas (eds.): Historiographies. Concepts et débats I. Paris: Galimard, pp. 468-476.

Schreibman, Susan/Siemens, Ray/Unsworth, John (eds.) (2004): Companion to Digital Humanities. Oxford: Blackwell. 